\title{
Time-Resolved EPR Study on Singlet-Fission Induced Quintet Generation and Subsequent Triplet Dissociation in TIPS-Phenyl-Tetracene Aggregates
}

\author{
Hiroki Nagashima ${ }^{1}$, Shuhei Kawaoka ${ }^{2}$, Yasunori Matsui ${ }^{2}$, Takashi Tachikawa ${ }^{1,3}$, \\ Hiroshi Ikeda $^{2^{*}}$, and Yasuhiro Kobori ${ }^{1,3^{* *}}$ \\ ${ }^{1}$ Laser Molecular Photoscience Laboratory, Molecular Photoscience Research \\ Center, Kobe University, 1-1 Rokkodaicho, Nada-ku, Kobe 657-8501, Japan \\ ${ }^{2}$ Department of Applied Chemistry, Graduate School of Engineering, Osaka Prefecture \\ University, 1-1 Gakuen-cho, Nakaku, Sakai, Osaka, 599-8531, Japan \\ ${ }^{3}$ Department of Chemistry, Graduate School of Science, Kobe University, 1-1 \\ Rokkodai-cho, Nada-ku, Kobe 657-8501, Japan \\ *ikeda@chem.osakafu-u.ac.jp \\ **ykobori@kitty.kobe-u.ac.jp
}

\begin{abstract}
The singlet fission (SF) is expected to be powerful tool for exceeding the theoretical limit of the power conversion efficiency on the organic solar cells. However, little is known on the intermolecular SF mechanism in the solid state. In the present study, we have investigated intermolecular SF in 2-phenyl-6,11-bis(triisopropylsilylethynyl)tetracene (TIPS-Ph-Tc) in frozen solutions using a time-resolved electron paramagnetic resonance measurement at a low temperature. SF-born quintet states (Q) and subsequent dissociations into two triplet states $(\mathrm{T}+\mathrm{T})$ were detected for a diluted solute concentration of $10^{-4} \mathrm{M}$ in dichloromethane. The microsecond triplet dissociation was found to occur due to the amorphous morphology in the frozen aggregate, suggesting that $\mathrm{Q}$ is generated as a trapped state. Furthermore, it was also suggested that the $\mathrm{T}+\mathrm{T}$ dissociation follows the $\mathrm{Q}$ generation due to a $\mathrm{T}-\mathrm{T}$ repulsion, whose energy were determined by the negative exchange couplings in the triplet pairs, (TT). Keywords: Organic photovoltaics, Singlet fission, Excited quintet, Triplet dissociation, EPR
\end{abstract}

\section{Introduction}

Much attention has been attracted on the development of organic photovoltaic devices as the next generation thin-film solar cells that can be lowcost, flexible and light [1]. However, the reported highest power conversion efficiency is $11 \%$, which is still lower than the efficiency of the recent lead halide perovskite solar cells. In this respect, the singlet fission (SF) process is highly expected to overcome the theoretical limit [2] of the solar cell efficiency (Shockley-Queisser limit) because of a potential of generating two charge-separations from the one high-energy photon. The SF reactions have been observed in limited organic molecules, i.e. tetracene $(\mathrm{Tc})$, pentacene $(\mathrm{Pn})$, hexacene, their derivatives, etc [3]. 6,13-Bis(triisopropyl- silylethynyl)pentacene (TIPS-Pn) and 6,11bis(triisopropylsilylethynyl)tetracene (TIPS- Tc) are representative molecules because of high stability under air, solubility to organic solvents, and high SF efficiencies (up to 200\%) [4], in comparison with the parent Pn or Tc.

Since applications of the SF to the organic solar cells are still challenging [5-10], it is desired to clarify the triplet separation dynamics and its energetics to obtain guidelines for designing highly efficient SF systems.

A simplified scheme (Fig. 1) for the conversion of a singlet exciton into two independent triplet states has been described as follows,

$$
\mathrm{S}_{0}+\mathrm{S}_{1} \rightleftarrows(\mathrm{TT}) \rightleftarrows \mathrm{T}_{1}+\mathrm{T}_{1}
$$


where $\mathrm{S}$ and $\mathrm{T}$ are singlet and triplet species, respectively. A symbol (TT) denotes a correlated triplet pair. The singlet exciton localized in one molecule (Frenkel exciton) interacts with the neighboring ground state molecule $\left(\mathrm{S}_{0}\right)$, generating the correlated intermolecular triplet pair with the singlet character, ${ }^{1}(\mathrm{TT})$. This triplet pair separates into independent triplets [11]. The SF requires that the energy of the excited singlet state is larger than the twice of the $T_{1}$ energy. Pn and their derivatives satisfy this requirement of $E\left(\mathrm{~S}_{1}\right)>2 E\left(\mathrm{~T}_{1}\right)$. In contrast, $\mathrm{Tc}$ and their derivatives would be classified to another case: the $S_{1}$ energy is slightly smaller than the twice of $T_{1}$ energy within the thermal energy of $k_{\mathrm{B}} T$ [12]. In this case, a geminate triplet-triplet pair may recombine to the $S_{1} S_{0}$ pair (triplet fusion, $T F$ ). This reverse process may follow the SF when the difference between $E\left(\mathrm{~S}_{1}\right)$ and $2 E\left(\mathrm{~T}_{1}\right)$ is comparable to $k_{\mathrm{B}} T$. When $E\left(\mathrm{~S}_{1}\right)<<2 E\left(\mathrm{~T}_{1}\right)$, separately produced two triplet species may encounter a leading to the $\mathrm{S}_{1}$ formation. This free pair reaction is known as the triplet-triplet annihilation (TTA). Detailed mechanisms of the SF, TF, and TTA are still under discussion on the organic semiconductors $[3,11,13]$.

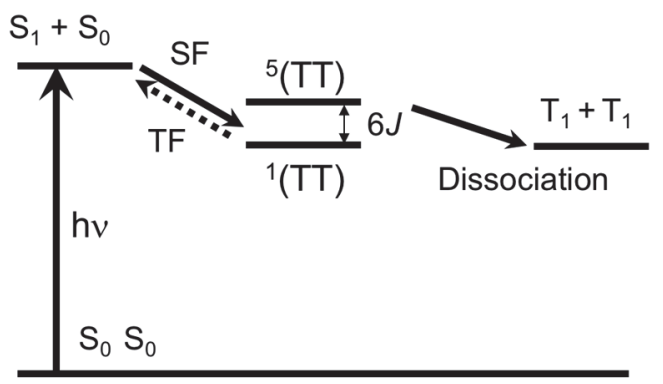

Fig. 1. Diagram of the singlet fission system. After photoexcitation, SF generates the quintet state ${ }^{5}(\mathrm{TT})$ of the two triplet pairs followed by singlet-quintet mixings. $J$ denotes the exchange coupling.

The electron paramagnetic resonance (EPR) spectroscopy has been a powerful tool to detect unpaired spins and investigate their electronic structures, geometries and dynamics [14-18]. Recently, the SF-born quintet state, corresponding to ${ }^{5}(\mathrm{TT})$, was observed by the time-resolved EPR (TREPR) method in thin films and macrocrystals of TIPS-Tc [19]. Intramolecular SF-born quintet states have also been reported on covalently linked dimers of TIPS-Pn [20,21]. Because the SF is spinallowed process, the SF-born quintet states are initially populated at a sublevel of $m_{s}=0$. Allowed EPR transitions from $\mid 0>$ to $\mid \pm 1>$ are thus detected in the EPR measurements $[19,20]$. The primary SF kinetics have been investigated on the covalently linked dimer molecules [20,22]. It is highly desired, however, that the primary SF dynamics is clarified for the intermolecular systems in the solid state, since such kinetics are informative for the practical applications to the organic solar cells. Although the intermolecular SF dynamics have been extensively investigated in the solid crystals and thin-films, the SF and triplet dissociation kinetics have not well been clarified in amorphous solids. In this study, we show that the quintet (TT)s are generated via SF in solute aggregates, accompanying exothermic microsecond triplet dissociation dynamics from the quintet state by using the TREPR measurements in frozen solutions for newly synthesized 2-phenyl6,11-bis(triisopropylsilylethynyl)tetracene (TIPSPh-Tc: Fig. 2).

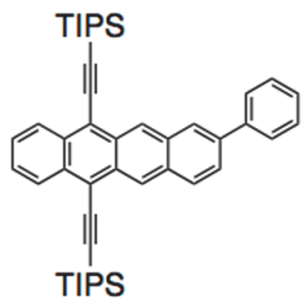

TIPS-Ph-Tc
TIPS:

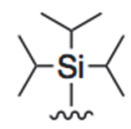

Fig. 2. Structure of TIPS-Ph-Tc.

\section{Experimental}

Synthesis of TIPS-Ph-Tc was performed as follows. In a $100 \mathrm{~mL}$ flask, a THF/water $(30 \mathrm{~mL} / 3$ $\mathrm{mL})$ solution of 2-bromo-6,11-bis(triisopropylsilylethynyl)tetracene $(340 \mathrm{mg}, 0.5$ mmol) [23], phenylboronic acid ( $83 \mathrm{mg}, 0.68 \mathrm{mmol}$ ), tetrakis(triphenylphosphine)palladium (110 mg, 0.1 $\mathrm{mmol})$, and $\mathrm{K}_{2} \mathrm{CO}_{3}(1.19 \mathrm{~g}, 8.6 \mathrm{mmol})$ was refluxed at $75{ }^{\circ} \mathrm{C}$ under $\mathrm{Ar}$ atmosphere for $48 \mathrm{~h}$ in the dark. After filtration through Celite pad, the mixture was extracted with $\mathrm{CHCl}_{3}(20 \mathrm{~mL} \times 3)$, dried over $\mathrm{Na}_{2} \mathrm{SO}_{4}$, and then concentrated under reduced pressure. After silica-gel column chromatography $(n$-hexane/EtOAc $=20 / 1)$, recrystallization from acetone gave TIPS-Ph-Tc (250 mg, $0.38 \mathrm{mmol}$, y. $76 \%)$ as dark red columns. mp $153-155{ }^{\circ} \mathrm{C} ;{ }^{1} \mathrm{H}$ NMR $\left(300 \mathrm{MHz}, \mathrm{CDCl}_{3}\right) \delta_{\text {ppm }} 1.27-1.43(\mathrm{~m}, 42 \mathrm{H})$, 7.41-7.45 (m, 1H), 7.52-7.57 (m, 4H), 7.77 (dd, $J$ $=9.0,1.4 \mathrm{~Hz}, 1 \mathrm{H}), 7.79-7.82(\mathrm{~m}, 2 \mathrm{H}), 8.10(\mathrm{~d}, J=$ $9.0 \mathrm{~Hz}, 1 \mathrm{H}), 8.18(\mathrm{~m}, 1 \mathrm{H}), 8.63(\mathrm{dd}, J=6.9,3.2 \mathrm{~Hz}$, 2H), $9.32(\mathrm{~s}, 1 \mathrm{H}), 9.35(\mathrm{~s}, 1 \mathrm{H}) ;{ }^{13} \mathrm{C} \mathrm{NMR}(75 \mathrm{MHz}$, $\left.\mathrm{CDCl}_{3}\right) \delta_{\text {ppm }} 11.9(3 \mathrm{C}+3 \mathrm{C}), 19.2(6 \mathrm{C}+6 \mathrm{C}), 104.18$, $104.21,106.1,106.2,118.8,118.9,126.1,126.4$, 126.6, 126.8, 126.9, 127.0, $127.6(2 \mathrm{C}), 127.7$ (2C), $127.9,129.2(2 \mathrm{C}), 129.5,130.7,130.9,131.6,132.6$, 
132.9, 133.0, 138.6, 141.2; IR (ATR) $v=2942,2863$, $2145,1462,1385,1040,881,756$, and $666 \mathrm{~cm}^{-1}$; LR-mass (FAB, NBA) $m / z$ calcd for $\left[\mathrm{C}_{46} \mathrm{H}_{56} \mathrm{Si}_{2}\right]^{+}=$ 664.39, found 664; Anal. Calcd for $\mathrm{C}_{46} \mathrm{H}_{56} \mathrm{Si}_{2}$ : C, 83.07; H, 8.49; Si, 8.45. Found: C, 82.76; H, 8.84.

TIPS-Ph-Tc samples were dissolved in various solvents such as dichloromethane $\left(\mathrm{CH}_{2} \mathrm{Cl}_{2}\right)$, hexane, 2-methyltetrahydrofuran (MTHF) and toluene with concentrations of 50 and/or $100 \mu \mathrm{M}$. These solutions were loaded into EPR quartz tubes and degassed by the freeze-pump-thaw cycles. The Xband TREPR measurements were carried out using a Bruker EMX system at $77 \mathrm{~K}$. Light excitations were performed by the second harmonics $(532 \mathrm{~nm})$ of a Nd:YAG laser (Continuum, Minilite II, fwhm $\sim 5 \mathrm{~ns}$ ) with a laser depolarizer.

\section{Results and discussion}

Figure 3a shows the TREPR spectra of TIPS-PhTc obtained by 532-nm Nd:YAG laser excitations of frozen $\mathrm{CH}_{2} \mathrm{Cl}_{2}$ solution $(0.1 \mathrm{mM})$ at $77 \mathrm{~K}$. Two pairs of peaks assigned to $\mathrm{Q}$ and $\mathrm{T}$ showing 15 and $50 \mathrm{mT}$ splittings, respectively, were observed. The separation of T-peaks are consistent with the zerofield splitting (ZFS) of the excited triplet state of TIPS-Tc [24]. The splitting of the Q-peaks are similar to the splitting by the quintet state of the TIPS-Tc pair in thin films [19]. The spin polarization exhibiting the microwave emission (E)/absorption (A) (i.e. E/A pattern) in the Q peaks are also similar to the reported pattern observed for the intermolecular TIPS-Tc pair in the thin films.

When a $0.1 \mathrm{mM}$ TIPS-Ph-Tc solution was frozen in MTHF, which is a good solvent to solve the substrate, no TREPR signal was observed (not shown). On the other hand, clear Q and T signals were obtained for the TIPS-Ph-Tc sample dissolved in $n$-hexane even with a lower concentration of 50 $\mu \mathrm{M}$. These findings suggest that solute aggregates are produced by freezing the solutions at $77 \mathrm{~K}$ when the poor solvents are employed, contributing to the intermolecular SF. Since the peak splitting in the Q signal is almost $1 / 3$ of the splitting of the T peaks in Fig. 3a, we thus concluded that the Q signals are originating from the allowed EPR transitions from $\mid 0>$ to $\mid \pm 1>$ among the five sublevels of $|0>| \pm 1>$, and $\mid \pm 2>$ in the excited quintet states of the strongly coupled triplet pairs ${ }^{5}(\mathrm{TT})$ generated by the intermolecular SF in the solute aggregates, as observed in the thin films [19]. From the delay time dependence of the TREPR spectrum in Fig. 3a, one can see that the outer E/A polarized $\mathrm{T}$ peaks are rising in accordance with the decrease in the inner
Q polarization in a microsecond region. This is in contrast to the previous TREPR report on the microcrystalline thin films of TIPS-Tc; the inner Q and outer $\mathrm{T}$ intensities were essentially in equilibrium for nanosecond ranges at the low temperature [19]. The present results in Fig. 3a thus denotes that the separations of the individual triplet states exothermally occur from ${ }^{5}(\mathrm{TT})$, as shown in Fig. 1.
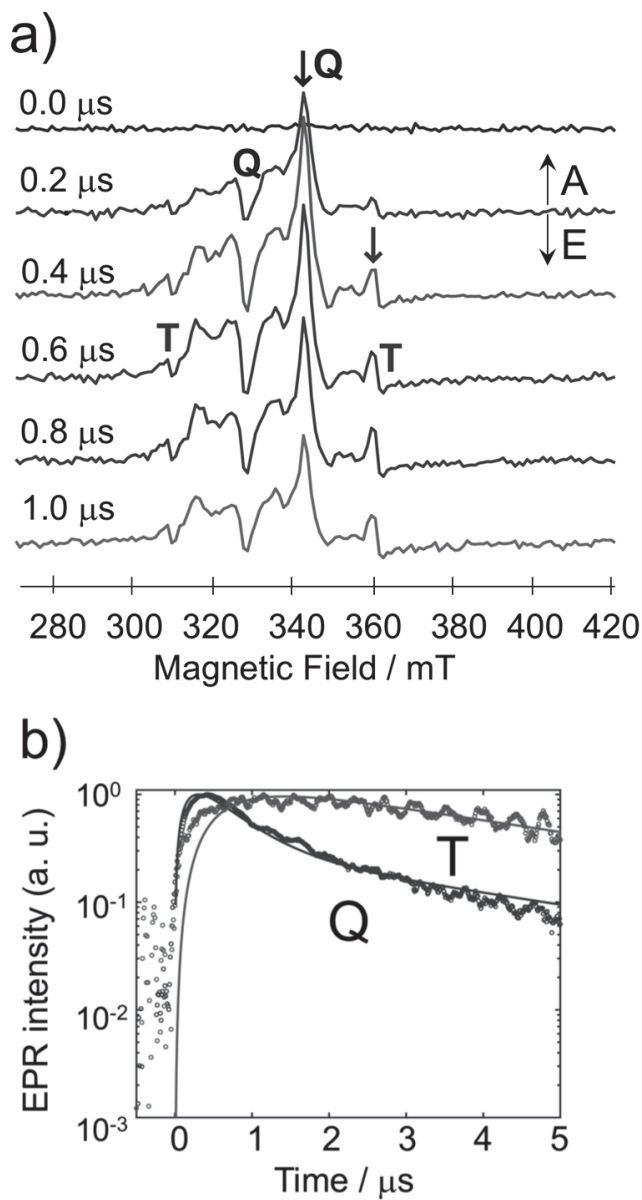

Fig. 3. (a) Delay time dependence of the TREPR spectrum of TIPS-Ph-Tc diluted in $\mathrm{CH}_{2} \mathrm{Cl}_{2}$ with a concentration of $0.1 \mathrm{mM}$ at $77 \mathrm{~K}$. (b) Time profiles of the TREPR intensities at $342 \mathrm{mT}$ for the quintet state (Q) of (TT) and at $359 \mathrm{mT}$ for the separated triplets (T).

To analyze the delay time dependence of the EPR signals, the time profiles (Fig. 3b) of the peak intensities shown by the dotted plots at $342 \mathrm{mT}$ in Q and at $359 \mathrm{mT}$ in $\mathrm{T}$ were reproduced by solving a coupled rate equation determined by the kinetics of ${ }^{5}(\mathrm{TT})$ and $\mathrm{T}_{1}+\mathrm{T}_{1}$. From eq. (1) the coupled equation is represented by the dissociation rate constant, $k_{\mathrm{QT}}$ for ${ }^{5}(\mathrm{TT}) \rightarrow \mathrm{T}_{1}+\mathrm{T}_{1}$, the geminate back-reaction rate constant, $k_{\mathrm{QT}}$ for $\mathrm{T}_{1}+\mathrm{T}_{1} \rightarrow{ }^{5}(\mathrm{TT})$, and the spinlattice relaxation rate constants of $k_{1 \mathrm{Q}}$ and $k_{1 \mathrm{~T}}$ for ${ }^{5}(\mathrm{TT})$ and $\mathrm{T}_{1}+\mathrm{T}_{1}$, respectively. The least-square 
fitting curves are shown by the lines for ${ }^{5}(\mathrm{TT})$ and $\mathrm{T}_{1}+\mathrm{T}_{1}$ in Fig. 3b. The resultant rate constants were obtained to be $k_{\mathrm{QT}}=0.53 \mu \mathrm{s}^{-1}, k_{\mathrm{TQ}}=0.30 \mu \mathrm{s}^{-1}, k_{1 \mathrm{Q}}=$ $0.63 \mu \mathrm{s}^{-1}$ and $k_{1 \mathrm{~T}}=0.29 \mu \mathrm{s}^{-1}$. From the dissociation and back reaction rates constants, the free energy change $\left(\Delta G_{\text {diss }}\right)$ for the triplet dissociation in Fig. 1 is estimated to be $\Delta G_{\text {diss }}=-3.6 \mathrm{meV}$ by $\Delta G_{\text {diss }}=-$ $k_{\mathrm{B}} \ln \left(k_{\mathrm{QT}} / k_{\mathrm{TQ}}\right)$. This energy is well consistent with $\Delta G_{\text {diss }}=-4.2 \mathrm{meV}$ obtained from the temperature dependence of the ratio of the quintet to triplet formation in the thin films of TIPS-Tc, representing that the dissociation energy would be an upper bound of the intermolecular spin-spin exchange coupling $(J)$ of (TT) in Fig. 1 [19]. The magnitude of $3 J\left(=\Delta G_{\text {diss }}\right)$ for (TT) in Fig. 1 is also coincident with the strongly coupled regime to form the quintet spin state, since the ZFS interaction in the present system is much weaker than the $J$ coupling[19]. The present microsecond dissociation dynamics in the frozen aggregate is however highly different from the quick equilibrium between ${ }^{5}(\mathrm{TT})$ and $\mathrm{T}_{1}+\mathrm{T}_{1}$ in the previously reported thin film of TIPS-Tc. This would be due to a disordered morphology formed by freezing the diluted solution in the present system, since the diffusion coefficients of the triplet excitons are known to be extremely smaller in amorphous environments than those in crystalline phases [25].

To check whether the geminate recombination (or $\mathrm{TF}$ ) occurs from ${ }^{5}(\mathrm{TT})$ to the $\mathrm{S}_{1} \mathrm{~S}_{0}$ pair in Fig. 1, we also measured the fluorescence decay (not shown) in the present frozen solution system using a streak scope with a picosecond light pulser. Although a double exponential decay profile was detected in the nanosecond time scale, we were unable to detect the TF-induced emission [26] in the submicrosecond region. This result and $\Delta G_{\text {diss }}<0$ obtained above suggest that the free energy $(G)$ of the $\mathrm{S}_{1} \mathrm{~S}_{0}$ pair, ${ }^{5}(\mathrm{TT})$ and $\mathrm{T}_{1}+\mathrm{T}_{1}$ obeys the relationship, $G\left(\mathrm{~S}_{1}\right)>$ $G\left[{ }^{5}(\mathrm{TT})\right]>G\left(\mathrm{~T}_{1}+\mathrm{T}_{1}\right)$, as shown in Fig. 1, indicating that the SF-requirement of $E\left(\mathrm{~S}_{1}\right)>2 E\left(\mathrm{~T}_{1}\right)$ is fulfilled in the present low-temperature aggregate.

In conclusion, by using the TREPR method (the 532-nm laser excitation, $77 \mathrm{~K}$ ), we have characterized the SF-induced $\mathrm{T}_{1}+\mathrm{T}_{1}$ dissociation dynamics and the exchange coupling of the strongly correlated triplet pair in the frozen aggregates of TIPS-Ph-Tc. It has been finally revealed that the microsecond triplet dissociation occurs due to the amorphous morphology in the solute aggregate which is simply produced by freezing the diluted solution, suggesting that ${ }^{5}(\mathrm{TT})$ is generated as a trapped state. However, the $\mathrm{T}+\mathrm{T}$ dissociation can follow the trap generation probably due to the repulsion, whose energy were determined by the negative $J$ coupling (Fig. 1) in (TT) even in the amorphous solid state. The present fundamental energetic and kinetic properties are highly informative for designing efficient energyconversion systems for the OPV devices.

\section{Acknowledgements}

This study was partially supported by JSPS KAKENHI grant (Nos. JP17H01265, JP18K14202, and JP18H01967). The work at Kobe University was supported by a Grant-in-Aid for Scientific Research (No. 17K19105 and 16H04097 to Y. Kobori) from the Ministry of Education, Culture, Sports, Science and Technology, Japan.

\section{References}

1. G. Yu, J. Gao, J. C. Hummelen, F. Wudl, and A. J. Heeger, Science, 270 (1995) 1789.

2. W. Shockley and H. J. Queisser, J. Appl. Phys., 32 (1961) 510.

3. M. B. Smith and J. Michl, Chem. Rev., 110 (2010) 6891.

4. B. J. Walker, A. J. Musser, D. Beljonne, and R. H. Friend, Nat. Chem., 5 (2013) 1019.

5. S. Lee, D. Hwang, S. I. Jung, and D. Kim, $J$. Phys. Chem. Lett., 8 (2017) 884.

6. B. Ehrler, M. W. B. Wilson, A. Rao, R. H. Friend, and N. C. Greenham, Nano Lett., 12 (2012) 1053.

7. S. Yoo, W. J. Potscavage, B. Domercq, S. H. Han, T. D. Li, S. C. Jones, R. Szoszkiewicz, D. Levi, E. Riedo, S. R. Marder, and B. Kippelen, Solid State Electron., 51 (2007) 1367.

8. B. Ehrler, B. J. Walker, M. L. Bohm, M. W. B. Wilson, Y. Vaynzof, R. H. Friend, and N. C. Greenham, Nat. Commun., 3 (2012) 1019.

9. Z. R. Hong, R. Lessmann, B. Maennig, Q. Huang, K. Harada, M. Riede, and K. Leo, J. Appl. Phys., 106 (2009) 064511.

10. A. K. Pandey, S. Dabos-Seignon, and J. M. Nunzi, Appl. Phys. Lett., 89 (2006) 113506.

11. N. Monahan and X. Y. Zhu, Annu. Rev. Phys. Chem., 66 (2015) 601.

12. P. M. Zimmerman, F. Bell, D. Casanova, and M. Head-Gordon, J. Am. Chem. Soc., 133 (2011) 19944.

13. M. B. Smith and J. Michl, Ann. Rev. Phys. Chem., 64 (2013) 361.

14. M. Hasegawa, H. Nagashima, R. Minobe, H. Mino, and Y. Kobori, J. Phys. Chem. Lett., 8 (2017) 1179. 
15. Y. Kobori, N. Ponomarenko, and J. R. Norris, $J$. Phys. Chem. C, 119 (2015) 8078.

16. H. Nagashima and H. Mino, Biochim. Biophys. Acta, 1827 (2013) 1165.

17. H. Nagashima and H. Mino, J. Phys. Chem. Lett., 8 (2017) 621.

18. M. Asada, H. Nagashima, F. H. M. Koua, J.-R. Shen, A. Kawamori, and H. Mino, Biochim. Biophys. Acta, 1827 (2013) 438.

19. L. R. Weiss, S. L. Bayliss, F. Kraffert, K. J. Thorley, J. E. Anthony, R. Bittl, R. H. Friend, A. Rao, N. C. Greenham, and J. Behrends, Nat. Phys., 13 (2016) 176.

20. M. J. Y. Tayebjee, S. N. Sanders, E. Kumarasamy, L. M. Campos, M. Y. Sfeir, and D. R. McCamey, Nat. Phys., 13 (2016) 182.

21. S. Lukman, K. Chen, J. M. Hodgkiss, D. H.
Turban, N. D. Hine, S. Dong, J. Wu, N. C. Greenham, and A. J. Musser, Nat. Commun., 7 (2016) 13622.

22. T. Sakuma, H. Sakai, Y. Araki, T. Mori, T. Wada, N. V. Tkachenko, and T. Hasobe, J. Phys. Chem. A, 120 (2016) 1867.

23. S. N. Sanders, E. Kumarasamy, A. B. Pun, M. L. Steigerwald, M. Y. Sfeir, and L. M. Campos, Angew. Chem. Int. Ed., 55 (2016) 3373.

24. S. L. Bayliss, K. J. Thorley, J. E. Anthony, H. Bouchiat, N. C. Greenham, and A. D. Chepelianskii, Phys. Rev. B, 92 (2015) 115432.

25. O. V. Mikhnenko, P. W. M. Blom, and T.-Q. Nguyen, Energ. Environ. Sci., 8 (2015) 1867.

26. G. B. Piland, J. J. Burdett, D. Kurunthu, and C. J. Bardeen, J. Phys. Chem. C, 117 (2013) 1224. 